\title{
Factores clave en la evaluación de la productividad: estudio de caso *
}

\author{
Key Factors in Productivity Assessment: A Case Study \\ Jorge Ariel Franco-López \\ Magister en Administración (MBA), Instituto Tecnológico Metropolitano, \\ Medellín-Colombia, jorgefranco@itm.edu.co \\ Julián Alberto Uribe-Gómez \\ Magister en Gestión Tecnológica, Instituto Tecnológico Metropolitano, \\ Medellín -Colombia, julianuribe@itm.edu.co \\ Sebastián Agudelo-Vallejo (iD \\ Tecnólogo en Administración, Instituto Tecnológico Metropolitano, \\ Medellín - Colombia, agudelo724@hotmail.com
}

\begin{abstract}
Cómo citar / How to cite
Franco-López, J. A., Uribe-Gómez, J. A., Agudelo-Vallejo, S. (2021). Factores clave en la evaluación de la productividad: estudio de caso. Revista CEA, v. 7, n. 15, e1800. https://doi.org/10.22430/24223182.1800

Recibido: 26 de abril de 2021

Aceptado: 6 de agosto de 2021
\end{abstract}

\section{Resumen}

La productividad es una condición inicial para obtener crecimiento económico, y para tener mejores condiciones de vida: se debe partir de ser eficientes y eficaces, siendo esta la combinación óptima de los recursos, pues, eficiencia más eficacia es igual a productividad. El objetivo de la investigación fue examinar las percepciones de los empleados en distintas organizaciones del sur del Valle de Aburrá: Envigado, Itagüí, Sabaneta, La Estrella y Caldas en el departamento de Antioquia (Colombia), frente a factores asociados con la productividad. Como metodología, se utilizó un sistema categorial que reunió 10 factores como determinantes de la productividad, el cual se construyó a partir de un rastreo bibliográfico. El enfoque de la investigación fue de tipo mixto. Para el trabajo de campo se aplicaron encuestas estructuradas con 10 preguntas cerradas en 17 organizaciones, utilizando la escala de Likert, obteniendo respuestas de 65 empleados. Posteriormente se tabularon los cuestionarios y se hizo el análisis respectivo para cada pregunta, donde se aplicó la estadística descriptiva y la matriz de correlación, arrojando como resultado que las categorías más decisivas en

\footnotetext{
* Este artículo se deriva del proyecto titulado «Factores clave en la evaluación de la productividad: estudio de caso» del programa Jóvenes Investigadores ITM y ha sido financiado con recursos del Instituto Tecnológico Metropolitano.
} 
la productividad son: variable laboral, articulación de políticas, infraestructura física, innovación, investigación y avance tecnológico. Se concluye que la motivación tiene la mayor cantidad de vínculos de correlacionales fuertemente lineales con los demás factores.

Palabras clave: comportamiento económico, productividad laboral, productividad en las organizaciones, organizaciones productivas, eficiencia de la producción.

Clasificación JEL: J24, P42.

\title{
Highlights
}

- La productividad es una condición inicial para obtener crecimiento económico y para tener mejores condiciones de vida.

- Los niveles de productividad varían porque las condiciones para obtener el rendimiento siempre serán diferentes.

- Existen cinco factores claves que determinan la productividad en las organizaciones: gestión del recurso humano, cultura organizacional, procesos productivos, estrategia de gestión organizacional y estrategia de desempeño.

- La productividad se convierte en un aspecto fundamental para todos los países y sus economías, especialmente en mercados de alta competencia.

- Ser productivos en un mercado cada vez más exigente y difícil como el actual, es la clave para permanecer y sobresalir; es una medida de la eficiencia de la producción.

\begin{abstract}
Organizational productivity is the basis to generate economic growth and better living conditions. An efficient and effective use of resources is an optimal combination because efficiency plus effectiveness equals productivity. This study examines the perceptions of employees, working at several organizations in the south of the Aburrá Valley (Envigado, Itagüí, Sabaneta, La Estrella, and Caldas in the Department of Antioquia, Colombia), of different factors associated with productivity. The methodology adopted here included a categorical system composed of 10 factors as determinants of productivity, which was designed based on a literature review. This study adopted a mixed-methods approach. A structured survey with 10 closed-ended questions and a Likert scale was answered by 65 employees at 17 organizations. Their answers were tabulated, and each question was analyzed using descriptive statistics and a correlation matrix. Based on the results, the categories that exert the greatest influence on organizational productivity are job stability, policy articulation, physical infrastructure, innovation, research, and technological progress. It is also concluded that motivation presents the highest number of strongly linear correlations with the other factors.
\end{abstract}

Keywords: Economic behavior, workforce productivity, organizational productivity, productive organizations, production efficiency.

JEL classification: J24, P42. 


\section{Highlights}

- Productivity is the basis to generate economic growth and better living conditions.

- Productivity levels vary because the conditions to make a profit are always different.

- Five key factors determine organizational productivity: human resource management, organizational culture, production processes, organizational management strategy, and performance management strategy.

- Productivity is essential for all countries and their economies, especially in highly competitive markets.

- Being productive in an increasingly demanding and difficult market such as the current one is the key to survive and stand out; it is a measure of production efficiency.

\section{INTRODUCCIÓN}

La productividad es un tema relevante que se ha identificado como un reto de las organizaciones (Smith, 2019; Sedaghat, 2013). Koontz et al. (2012, p. 550) mencionan que

En el siglo XXI, la productividad es sin duda, una de las preocupaciones más importantes de los administradores en muchas partes del mundo, hasta en Japón, país admirado por sus mejoras en la productividad, están preocupados por mantenerse competitivos en el mercado mundial

A su vez, es una condición para el crecimiento económico (Alonso Villa y Juste Carrión, 2018; Bušelić y Pavlišić, 2016); ahora bien, para tener mejores condiciones de vida se debe partir de ser eficientes y eficaces y contar con otros aspectos del desarrollo social, tales como: educación, servicios públicos, habita digna, conectividad, comunicaciones, etc. (Quinde Rosales et al., 2020). En esencia, la productividad es la combinación óptima de los recursos y factor clave para competir en los mercados.

Los niveles de productividad varían porque las condiciones para obtener el rendimiento siempre serán diferentes; por tanto, el interrogante que motivó a realizar esta investigación fue, ¿qué categorías afectan la productividad en un contexto organizacional determinado? De este modo, el objetivo fue examinar las percepciones de los empleados en 17 organizaciones de los municipios que conforman el sur del Valle de Aburrá, frente a factores asociados con la productividad, donde, además, fueron aplicadas 10 variables, o categorías en cuestión, a dichas organizaciones.

En la vasta literatura sobre el concepto productividad, se menciona el trabajo de Kamble y Wankhade (2017), quienes aducen que existen cinco factores claves que determinan la productividad en las organizaciones: gestión del recurso humano, cultura organizacional, procesos productivos, estrategia de gestión organizacional y estrategia de desempeño. Estas consideraciones indican en forma inicial un derrotero investigativo, en el cual, después de una búsqueda bibliográfica, se concretaron diez factores que conforman el sistema categorial, que modifican la productividad de las organizaciones seleccionadas, ubicadas en el área sur de Medellín, sector con alta concentración en la producción local: 1) aspectos sociales, 2) remuneración salarial, 3) formación del personal, 4) articulación de políticas y apoyo gubernamental o la relación organización - Estado, 5) infraestructura física, 6) lo laboral que conlleva al mercado de trabajo, 7) la motivación de quienes están en los procesos 
productivos y organizacionales, 8) la capacidad que tiene la organización para adaptarse al cambio, 9) innovación, investigación y avance tecnológico, 10) medio ambiente.

El abordaje teórico recogió varias propuestas sintetizadas en diferentes áreas. Para algunos pensadores no existe una teoría de la productividad en forma directa, Adam Smith (1776), David Ricardo (1817), citados por Fau (2000); Taylor (1878), citado por (Mera, 2017); sin embargo, en última instancia, las propuestas apuntan a lograr un bienestar en la sociedad, lo cual solo se alcanza, en primer lugar, obteniendo niveles elevados de productividad. En lo metodológico, para conseguir información primaria, se hizo a través de un formulario con un contenido de 10 preguntas tipo escala Likert, aplicado a 65 empleados en organizaciones con distinto objeto social en la región. Para el análisis de la información se utilizó la estadística descriptiva y la matriz de correlación.

\section{MARCO TEÓRICO}

En globalización, la productividad se convierte en un aspecto fundamental para todos los países y sus economías, especialmente en mercados de alta competencia (Valli y Saccone, 2015). Ser productivos en un mercado cada vez más exigente y difícil como el actual, es la clave para permanecer y sobresalir, es una medida de la eficiencia de la producción. Por productividad, indican Laborde y Veiga (2011), se entiende la relación entre lo producido y lo que es requerido para producir; por su parte, el Centro de Ciencia y Tecnología de Antioquia (s.f.), indicó que productividad es la búsqueda hacia el mejoramiento de todo cuanto existe, es la convicción en los procesos donde hoy se deben hacer mejor que ayer, y mañana mejor que hoy. "Mientras más productiva sea una economía, tanto mayor será su competitividad en el mercado internacional y menor su tasa de desempleo» (Medina Fernández de Soto, 2009). De igual forma lo demuestra el trabajo de Vázquez López (2021) con respecto de la productividad labor y la competitividad del sector manufacturero mexicano.

Este concepto difiere de acuerdo con cada contexto o lugar. Por ejemplo, en el aspecto comparativo en América Latina, la situación no es favorable con respecto a otras regiones como Norteamérica, Europa y Asia (Alvarez, 2015). Allí hay una relación estrecha entre productividad y crecimiento económico, por ello «EI PIB y la productividad en América Latina, han sido lentos y decepcionantes en las últimas tres décadas» (Ros, 2014), y, como lo indica Álvarez Pinilla (2001), productividad es el número de unidades output producidas por recursos o factores empleadas en la producción; es decir, en América Latina la relación entre producto y recursos no es la mejor, el producto obtenido no compensa la relación con los factores ocupados. En esta región, la baja productividad lleva a un bajo crecimiento y a una lenta acumulación de capital (Hofman et al., 2017). Cimoli et al. (2005, p. 9-10) mencionan que

Las economías latinoamericanas sufren un marcado rezago con respecto a un grupo de economías desarrolladas tomadas como referencia, tanto en lo que se refiere a participación de estos sectores como en lo relativo a la evolución de los niveles de productividad

En Villalobos Valencia et al. (2021) destacan, en un análisis de contabilidad del crecimiento de Suramérica, cómo la productividad no ha sido un factor determinante en el crecimiento. Por su parte, el trabajo de Villegas Pocaterra et al. (2020) indica la importancia que tiene la productividad y la eficiencia de los factores productivos en la explicación de los niveles de ingreso, lo cual hace la 
diferencia con otros países, por su parte, esta deficiencia de factores en la región suramericana es uno de los muchos factores que explican las relaciones de atraso con respecto de otras regiones en el mundo.

En el caso de Colombia, sus resultados en competitividad distan, comparativamente, de los estándares internacionales. Según Clavijo (2003), entre 1950-1980 la productividad multifactorial en Colombia fue de $0.8 \%$ anual, y cayó a un promedio de $-1.5 \%$ entre los años 1981-2002. Esto evidencia un desempeño mediocre de la productividad nacional comparado con los desempeños de las economías asiáticas, que fue del 1.3\% en Taiwán, Corea del Sur, Singapur y Hong Kong, y el 1.2\% de Indonesia y Malasia, jugando un papel importante la educación como estrategia de política económica. Existen múltiples aspectos que contribuyen a esta situación, desde condiciones de orden antropológico, sociológico, económico, educativo, y otros más que, al conjugarse, representan bajos niveles de productividad. «Mientras que la acumulación de factores en América Latina ha estado en línea con el resto del mundo, lo que diferencia el crecimiento económico de América Latina es su menor crecimiento de la productividad total de los factores» (Gómez Muñoz et al., 2019).

La productividad es una primera condición para lograr mejores estándares de vida, la cual, obviamente, se debe complementar con aspectos de competitividad y todas las políticas gubernamentales para un mejor crecimiento y desarrollo. El Estado colo0mbiano está interesado en mejorar la productividad, por ello «desde 2008, con el diseño del Conpes 35273, se aprobó para Colombia la Política Nacional de Competitividad y Productividad, la cual está en pleno proceso de aplicación y de la que se espera obtener los mejores resultados» (Tejedor, 2013). Ahora, lo anterior no es suficiente para pensar que todo depende de decisiones de tipo estatal, sino que es necesario un relevo generacional con nuevas perspectivas de vida que tengan otras dinámicas formadas desde diferentes niveles de socialización, pues el problema de mejorar la productividad va desde aspectos tangenciales, pero también vivenciales.

Desde lo conceptual, para el desarrollo del sistema categorial argumentado desde la introducción, evidencia la literatura, como en Bragg et al. (2021), la importancia que tiene la investigación para aumentar la productividad. El estudio fue desarrollado para construir equipos de investigación productivos. Para lograr mejores condiciones de vida se deben hacer serios ajustes a la estructura productiva para tener más productividad, ser más competente, más rendidores en la tarea, como lo indican Hinojo Lucena et al. (2020). Una tarea importante es adaptar el sistema educativo a la iniciativa gubernamental, se debe incursionar en propuestas motivadas por la innovación y los modelos I+D+i (Krizanic et al., 2021; Kim y Park, 2018). La investigación en sectores estratégicos de alto valor agregado, debe ser la respuesta a las nuevas dinámicas del crecimiento y desarrollo. Los paradigmas deben cambiarse o ajustarse. «El aumento en productividad tiene fuentes distintas en los países desarrollados, en comparación con los países en desarrollo. Los primeros se enfocan en investigación y desarrollo para producir innovaciones, mientras los otros se concentran en modificar su estructura productiva» (Maldonado y Sánchez, 2012). Esto es un aspecto clave: la estructura productiva modificada por procesos de investigación que generan innovación, «las empresas especializadas en I+D y marketing tienen niveles de productividad significativamente más altos» (de Vries et al., 2021). Por su parte se destaca que «las estrategias de innovación tienen efectos positivos sobre la productividad y el impacto ambiental de las empresas manufactureras donde las grandes empresas tienen efectos más significativos en la relación entre las estrategias de innovación y la productividad» (Seclen-Luna et al., 2021). 
Otro aspecto a tener en cuenta es la relación entre el salario real y la productividad. El Banco Interamericano de Desarrollo (2010), citado por Vera y Vera (2021), establece que, con una menor productividad, se fija un salario real más bajo; en ese sentido los gobiernos, para tratar de mantener el control social, deben, a través de la política económica, sostener el nivel de vida de los trabajadores con programas sociales. En la teoría económica neoclásica relaciona los factores o recursos con su remuneración; en relación con eso, el salario real se ajusta a los cambios de la productividad marginal del trabajo. Hay una relación directa entre las dos variables. Baptista (2005) hace un estudio de la economía venezolana relacionando el salario real con respecto de la productividad entre 1950 y 2004. El trabajo de Marquetti (2004) también da cuenta de la relación entre los salarios reales y la productividad en Estados Unidos. "Los salarios tienden a ser más altos como resultado de una mayor productividad asociada con las economías de aglomeración, pero esto último no se traduce necesariamente en un aumento del empleo» (Partridge et al., 2021, pág 4).

La estabilidad laboral, entendiéndose como la permanencia que tiene un trabajador en las actividades laborales, también es un aspecto que genera productividad y «reducción de la rotación de personal, el aumento del espíritu de equipo en la empresa, etc., suelen generar un aumento de la productividad laboral» (Bril et al., 2021). Desde esta perspectiva, la estabilidad en los puestos de trabajo suele repercutir en productividad y en una motivación para el trabajador, lo que es conveniente desde el punto de vista de la organización porque permite obtener niveles de especialización en las tareas, con lo cual contribuye a niveles de eficiencia y eficacia, y, por otra parte, esa estabilidad laboral le genera al trabajador mejores condiciones de vida. Cabe mencionar también la productividad con relación a los aspectos sociales diferenciado entre sectores productivos. Aquí destaca el trabajo de Vestby et al. (2021), quien concibe que la brecha de productividad existente entre el sector tradicional y el moderno genera conflictos a través de la diferenciación salarial, y con ello todo el deterioro social que se ve reflejado en el aumento de los índices de inseguridad que están latentes ante la caída de los ingresos de un sector tradicional, y que embarga, especialmente, las economías atrasadas.

El medio ambiente es un tema importante como objeto de investigación en la productividad. Algunas de ellas destacan lo anterior. Se menciona el trabajo de Khan et al. (2021), en el cual indican la importancia que tiene el agua en ciertos procesos productivos en Pakistán, destacando que solo así se logra obtener productividad en la región; por su parte, Zhang y Wu (2021) indica que, para el caso de China, una economía en crecimiento y con una dinámica única que la han hecho ser la de mayor producción del mundo, es vital aplicar tecnología inteligente marcada por factores ecológicos verdes para tener un impacto favorable en la productividad.

La investigación de Bergston et al. (2021) detalla cómo como la infraestructura locativa de la organización, denominada lugares basados en actividades ( $A B W$ ), genera un espacio propicio donde se obtiene mayores niveles de productividad. El estudio detalló que el modelo ABW facilita la flexibilidad de las personas en el trabajo, el uso de espacios óptimos para diferenciar tareas laborales y permite que los empleados puedan tener buen movimiento, lo que afecta positivamente la productividad en la organización. El trabajo de Jensen y van der Voordt (2020) evidencia que en la administración de instalaciones y en bienes corporativos, se puede contribuir más directamente a una mayor productividad cuando se haga una mejor distribución del espacio que respalde las actividades en la organización. En cuanto al tema del espacio en la ciudad, Peralta et al. (2019) 
destaca que el poco avance en la infraestructura en vías, o en los sistemas de transporte ha hecho colapsar el flujo de los vehículos, aumentando los tiempos de viaje, afectando la calidad de vida y reduciendo la productividad.

Otra faceta dentro de los estudios y quizás poco explorado en la literatura, es la relación que tiene la productividad con la motivación. El trabajo de McNamara et al. (2021) indica la dicotomía entre premio y castigo. Este último tiene un impacto negativo en la productividad, al reducir la motivación intrínseca y aumentar la rotación de empleados. El incentivo para el trabajador es una mejor remuneración salarial, y, a su vez para el empresario, es obtener una mayor productividad, porque esto coloca a la organización en mejor nivel de competencia en el mercado: «destacamos la contribución de las teorías de la motivación y del aprendizaje a la investigación, y la relación entre el castigo y la productividad en la economía y la gestión del trabajo» (p. 2).

El trabajo de investigación presentado delimita el estudio de la productividad en la zona sur del área metropolitana del Valle de Aburrá, la cual tiene una superficie de $1152 \mathrm{~km}^{2}$, dentro de los cuales se incluyen diez municipios o localidades. El Valle de Aburrá constituye la aglomeración urbana de mayor importancia del occidente colombiano, con 3449049 habitantes que representa algo más del 5\% de la población total nacional. Medellín es la segunda ciudad del sistema urbano del país, y alberga el $40 \%$ del total de habitantes del departamento de Antioquia. El 17.7\% de la población se encuentra en los cinco municipios de la zona sur del Valle de Aburrá (Caldas, Envigado, Itagüí, La Estrella y Sabaneta) (Área Metropolitana del Valle de Aburrá, 2010).

Medellín, denominada otrora "la ciudad industrial de Colombia», alrededor de las tres últimas décadas ha venido cambiando su estructura productiva. Hoy, sus mayores actividades productivas las representa el sector terciario o deservicios. Según el Observatorio Aburrá Sur (2016), en la región las empresas de comercio son el 56.6\%; servicios 21.15\%; manufactura $14.18 \%$; transporte y telecomunicaciones 3.23\% (distribuidas en los municipios de Medellín, Itagüí, Envigado, Sabaneta, La Estrella y Caldas). Esta área, considerada clave por ser el corredor desde y hacia el sur del país, viene cambiando su vocación productiva; la mayor cantidad de organizaciones son comerciales, con un fenómeno particular: la desindustrialización de la zona. «El sur del Valle de Aburrá se está desindustrializando, por lo que hay una migración hacia la actividad de servicios, para generar nuevas apuestas sectoriales con mayor dinámica económica» (Arias, 2011). Otros aspectos importantes del área metropolitana son el agotamiento del suelo, altos problemas de movilidad y seguridad. La Tabla 1 evidencia, según diferentes autores, la búsqueda como soporte del concepto productividad desde la contribución explícita e implícita a partir de lo económico, lo administrativo y en calidad. 
Tabla 1. Consideraciones teóricas sobre el concepto de productividad en diferentes áreas del conocimiento Table 1. Theoretical considerations on the concept of productivity in different knowledge fields

\begin{tabular}{|c|c|}
\hline Autor & Aporte en el área económica \\
\hline William Petty & Los trabajadores como factor para mejorar la productividad. \\
\hline Adam Smith & $\begin{array}{l}\text { Para aumentar la productividad y los ingresos de los trabajadores, se hace por medio de la } \\
\text { división del trabajo, al dedicarse solo a una labor (especialización). "La diversificación } \\
\text { productiva (en sus palabras, la “división del trabajo") y la capacidad de compra de la población } \\
\text { (en sus palabras, la “extensión del mercado") son los principales motores de la productividad y } \\
\text { del crecimiento económico en el sistema capitalista» (Ortiz et al., 2019, p. 101). }\end{array}$ \\
\hline $\begin{array}{l}\text { Thomas } \\
\text { Malthus }\end{array}$ & $\begin{array}{l}\text { La educación mejora al hombre y a la productividad. Es un medio, pero no el fin. «mejora al } \\
\text { hombre, pero no para crear riqueza» (Salcines y Freire, 2010). }\end{array}$ \\
\hline Robert Owen & $\begin{array}{l}\text { «(...) a partir de un trato más considerado hacia los trabajadores, como parte de un mejor } \\
\text { incentivo para su productividad» (Santos, 2002; Mantoux, } 1962 \text { citados por Jurado Jurado, } \\
\text { 2015). }\end{array}$ \\
\hline David Ricardo & Incluyó variables en sus explicaciones: salarios, y las tasas de cambio. \\
\hline Henri Fayol & La productividad debe estar presente dentro de todo el proceso administrativo. \\
\hline $\begin{array}{l}\text { Frederick } \\
\text { Winslow } \\
\text { Taylor }\end{array}$ & $\begin{array}{c}\text { «El Taylorismo es acertado y responde al aumento de la productividad» (Mayol, 2013). El salario } \\
\text { debe fijarse en función de la cantidad de producto elaborado como incentivo para aumentar la } \\
\text { productividad. }\end{array}$ \\
\hline Frank Gilbreth & $\begin{array}{l}\text { Movimientos en las tareas productivas, buscaba cómo disminuir los tiempos para ser más } \\
\text { eficiente. La ergonomía puesta al servicio de la productividad. }\end{array}$ \\
\hline $\begin{array}{l}\text { Henry L. } \\
\text { Gantt }\end{array}$ & $\begin{array}{l}\text { Trabajó inicialmente la propuesta taylorista sobre los tiempos en función de la producción, } \\
\text { luego migró hacia aspectos humanísticos, se enfocó en el aspecto salarial y de incentivos. }\end{array}$ \\
\hline Mary Parker & Enfoque entre relaciones humanas al mejoramiento de la productividad. \\
\hline Elton Mayo & $\begin{array}{l}\text { Hizo aportes desde lo humano lo que permite a la organización mejorar los aspectos de la } \\
\text { productividad, consideró al trabajador como eje fundamental de la producción, el «capital } \\
\text { humano». }\end{array}$ \\
\hline $\begin{array}{l}\text { Douglas } \\
\text { McGregor }\end{array}$ & $\begin{array}{c}\text { En la teoría, el trabajador disfruta de la actividad laboral. Busca en el trabajo gratificaciones de } \\
\text { orden superior, es ambicioso y está dispuestos a asumir responsabilidades, obteniendo } \\
\text { mayores niveles de productividad. }\end{array}$ \\
\hline $\begin{array}{l}\text { Abraham } \\
\text { Maslow }\end{array}$ & $\begin{array}{l}\text { No hay una teoría de la productividad, sin embargo, al analizar la propuesta se puede } \\
\text { considerar que las necesidades expuestas en la pirámide de las necesidades, muestra la } \\
\text { posibilidad que tiene un trabajador para ser productivo, «Estimaba que las necesidades } \\
\text { humanas tienen un acomodo jerárquico que asciende desde las inferiores hasta las superiores» } \\
\text { (Koontz et al., 2012). }\end{array}$ \\
\hline $\begin{array}{l}\text { Peter F. } \\
\text { Drucker }\end{array}$ & $\begin{array}{l}\text { La producción tiene otro recurso, el conocimiento. «Ahora tenemos ante nosotros otra } \\
\text { transformación pendiente, la productividad de los trabajadores no manuales, los del saber, que } \\
\text { consistirá en la aplicación del saber al saber.» (Sánchez Murillo, 2006). }\end{array}$ \\
\hline Autor & Aporte en el área de la calidad \\
\hline $\begin{array}{l}\text { W.Edwards } \\
\text { Deming }\end{array}$ & $\begin{array}{l}\text { Incide en los aspectos productivos por medio del enfoque sobre calidad, plantea el ciclo PHVA } \\
\text { (planificar, hacer, verificar y actuar.) como estrategia de mejora continua. }\end{array}$ \\
\hline $\begin{array}{c}\text { Joseph Moses } \\
\text { Juran }\end{array}$ & $\begin{array}{l}\text { Trilogía de la calidad: planificación, control y mejoramiento. La revolución de la calidad tuvo } \\
\text { como principios la implicación de los directivos, la capacitación de todos los empleados y la } \\
\text { participación de la mano de obra en la toma de decisiones. }\end{array}$ \\
\hline
\end{tabular}




\section{METODOLOGÍA}

La investigación desarrollada se clasifica como mixta, «representan el más alto grado de integración o combinación entre los enfoques cualitativo y cuantitativo. Ambos se entremezclan o combinan en todo el proceso de investigación» (Pereira Pérez, 2011). Se parte en la creación de un sistema categorial que pudiese ser evaluado siguiendo la metodología indicada para investigaciones de tipo cualitativa, al considerar que la información obtenida en cada organización era la percepción frente a la temática de la productividad que cada persona encuestada tiene al respecto. Los aspectos metodológicos pueden observarse en la Figura 1. Lo anterior implica, en un primer momento, lo que la metodología de Galeano (2004) denomina como exploratorio, la cual se hizo para responder a las asociaciones de las variables o categorías potenciales sobre la medida de la productividad en la organización; un segundo momento, llamado focalización, se efectúa para obtener los datos con base en encuestas sobre cómo dichas variables afectan efectivamente la productividad; y un tercer momento de profundización, donde se analizan los datos a través de estadísticas básicas y un modelo de correlación, lo cual coloca en evidencia el análisis cuantitativo como soporte para aceptar o rechazar las consideraciones teóricas mencionadas al inicio.

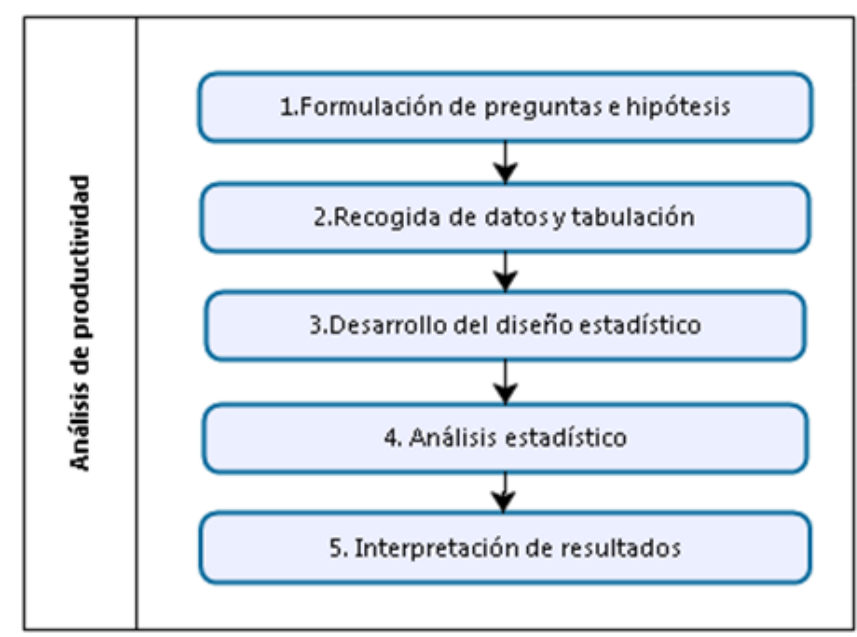

Figura 1. Estructura del método de investigación llevado a cabo

Figure 1. Structure of the research method applied in this study Fuente: elaboración propia.

Indica Galeano (2004), sobre la investigación cualitativa, que es aquella que se hace a los seres humanos, porque son capaces de pensar y entender una realidad que viven; no se trata de explicar, pero sí de entender una realidad particular, que al generalizarse se vuelve un producto social. Ahora, al aplicar la productividad a diferentes contextos sociales, como en Ayoví Ramírez y La Serna Palomino (2021), donde al considerar el alfa de Cronbach la correlación de Spearman y el modelo de Wright de senderos y rutas, logran esclarecer el impacto de la gestión de la innovación y la gestión estratégica en la productividad de las organizaciones del sector tecnológico y de la comunicación en Ecuador. Otro caso es el realizado por Delfín Ortega y Navarro Chávez (2020) con el estudio del análisis de la frontera estocástica donde precisan la productividad de los puertos de Shanghái y el Callao en Perú. En Guzmán Soria et al. (2020) aplican una función de producción no homogénea (NHPF, por sus siglas en inglés) doble logarítmica para estimar la eficiencia y productividad de la mipymes de México. 
El constructo categorial se logró mediante un rastreo bibliográfico, el cual arrojó 10 factores claves que dieron las pautas para el análisis (Gori Maia y Sakamoto, 2018; Quintero Arango y Betancur Arias, 2018; Vera, 2009). Estos factores, con su respectiva sigla, se pueden observar en la Tabla 2.

Tabla 2. Factores clave para la evaluación de la productividad

Table 2. Key factors for productivity assessment

\begin{tabular}{ccl}
\hline Factor & Sigla & \multicolumn{1}{c}{ Hipótesis para analizar } \\
\hline Aspectos sociales & Soc & $\begin{array}{l}\text { ¿El entorno social en el cual se encuentra la organización } \\
\text { tiene seguridad? }\end{array}$ \\
\hline Remuneración salarial & Sal & $\begin{array}{l}\text { ¿Cómo son los salarios que paga la organización a sus } \\
\text { empleados y trabajadores? }\end{array}$ \\
\hline $\begin{array}{c}\text { Frticulación de políticas y } \\
\text { apoyo gubernamental }\end{array}$ & For & $\begin{array}{l}\text { ¿Hay procesos de capacitación de la organización con sus } \\
\text { trabajadores? }\end{array}$ \\
\hline Infraestructura física & IEF & $\begin{array}{l}\text { ¿Cómo es la comunicación entre los trabajadores y los } \\
\text { jefes? }\end{array}$ \\
\hline Laboral & Lab & $\begin{array}{l}\text { ¿La organización cuenta con infraestructura física para el } \\
\text { cumplimiento de sus actividades? }\end{array}$ \\
\hline Motivación & Mot organización brinda estabilidad laboral con sus \\
trabajadores?
\end{tabular}

Aplicando una metodología de investigación experimental cuantitativa, se estableció la ruta para obtener el trabajo de campo. Como instrumento para la toma de información, se recogió una muestra de 17 organizaciones del sur del área metropolitana del Valle de Aburrá. La Tabla 3 muestra las organizaciones encuestadas por sectores económicos en la región indicada y una semblanza de cada una de ellas.

La Tabla 3 muestra las actividades en 17 organizaciones con diferente objeto social, las cuales fueron abordadas en la región sur del área metropolitana del Valle de Aburrá con el ánimo de obtener información. Para la selección de la muestra se utilizó la técnica de muestreo no probabilístico por conveniencia (de la Cuesta González et al., 2015), buscando la máxima heterogeneidad posible en los diferentes tipos de sectores empresariales encuestados y sus actores. Parte de la metodología se enfocó en organizaciones activas y que fueran representativas en el mercado local y regional. 
Tabla 3. Tipos de organizaciones encuestadas en el sur de área metropolitana de Medellín Table 3. Types of organizations where respondents work (in the south of the metropolitan area of Medellín)

\begin{tabular}{cc}
\hline Organización & Actividades que desarrolla \\
\hline 1 & Fabricación y transformación de lámina acrílica. Genera soluciones especiales en acrílico. \\
\hline 2 & Salas, cocinas, comedores y exteriores con sillas, taburetes y butacas. \\
\hline 3 & $\begin{array}{c}\text { Organización dedicada a la prestación de servicios de catering y logística de todo tipo de } \\
\text { eventos sociales y empresariales. }\end{array}$ \\
\hline 4 & $\begin{array}{c}\text { Compañía global de alimentos que busca cautivar al consumidor a través de la innovación y } \\
\text { el sabor de sus productos. }\end{array}$ \\
\hline 5 & $\begin{array}{c}\text { Operador logístico para eventos de carácter público y privado. Organizador de ferias, } \\
\text { convenciones, seminarios, conciertos, campañas de marketing digital y producciones a } \\
\text { gran escala. }\end{array}$ \\
\hline 6 & Marca colombiana de tradición, productora de alimentos. \\
\hline 7 & Encuentra todos tus productos para el hogar. \\
\hline 8 & Industria de las bebidas no alcohólicas en Colombia \\
\hline 10 & Portafolio más completo en América Latina en almacenamiento de energía. \\
\hline 11 & Solución de empaques para la industria cosmética, farmacéutica, aseo y hogar. \\
\hline 12 & Fabricación de artículos de plástico. \\
\hline 13 & Llanta, mecánica automotriz, lubricación. \\
\hline 14 & Juntos la vida sabe mejor, productos para el consumo. \\
\hline 15 & Compra de equipos y la construcción en procesos productivos, con la intención de fabricar \\
& productos a partir de granos. \\
\hline 16 & El outlet de las marcas originales. \\
\hline 17 & Fuente: elaboración propia. \\
\hline
\end{tabular}

El análisis de datos exploratorios en gran cantidad de proyectos de investigación implica examinar la correlación entre variables (Bruce et al., 2020) con el fin de conocer la asociación positiva y negativa entre las variables que reflejan la productividad y sus posibles efectos lineales. En este caso, inicialmente se utiliza un análisis descriptivo, que incluye conocer el comportamiento de las estadísticas básicas para cada una de las variables estudiadas; igualmente, se hace uso de la matriz de correlación, la cual, en términos generales, implica la creación de una tabla donde las variables son presentadas en filas y columnas, y donde las celdas muestran los valores de las correlaciones entre variables (Dangeti, 2017).

Con un formato de encuesta presencial individual, aplicada a 65 empleados de las 17 organizaciones, con 10 preguntas cerradas estructuradas que constaban de criterios de valoración, como las presentadas en la Tabla 2, se logró el conjunto de variables ordinales utilizando la escala de Likert. Los datos fueron recolectados en el año 2019 mediante la encuesta aplicada en horario laboral, donde cada empleado pertenecía a diferentes áreas de la organización, desde el nivel estratégico hasta el operativo. En los análisis de los hallazgos para la encuesta, la tabulación, gráficos y reportes estadísticos se utilizó el Microsoft Excel y Python 3.7. 


\section{RESULTADOS}

Se recurrió a un análisis descriptivo para encontrar las características de mayor interés para la medición de la productividad. La Figura 2 presenta el comportamiento de los resultados de la encuesta para cada una de las 10 preguntas realizadas, agrupadas por cada sector empresarial (industrial, comercial y de servicios) en escala Likert de medida.

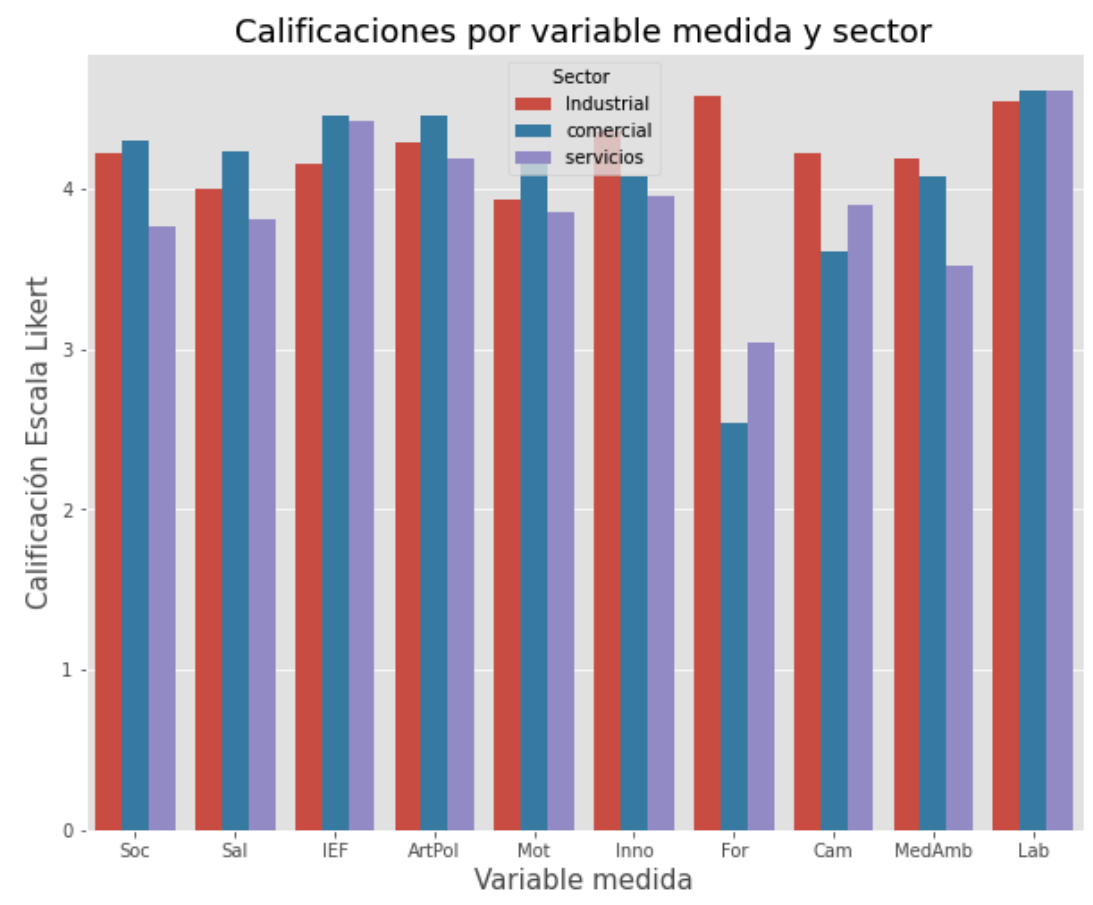

Figura 2. Resultados para cada pregunta de la encuesta

Figure 2. Results of each item in the survey Fuente: elaboración propia.

La Figura 3 muestra las variables agrupadas por el sector empresarial; en general, el sector industrial tiene mejores calificaciones en la escala de medida, en las cuales la variable Formación de personal y Laboral tienen las mejores calificaciones. Contrario para los sectores comercial y de servicios, la variable Formación de personal tiene las calificaciones más bajas y, en términos generales, la variable Laboral tiene mejor calificación entre todos los sectores.

La Tabla 4 presenta el resumen de las estadísticas básicas para cada una de las variables estudiadas que influyen sobre la productividad, según la literatura consultada. La mayor parte de los resultados rondan, en promedio, un puntaje de 4; es importante anotar que existe una alta dispersión en los resultados para algunas variables como motivación, innovación, investigación y avance tecnológico, formación y cambio, esto debido a su alto coeficiente de variación que afecta la productividad, tal como lo expresan Paixão et al. (2020), los cuales oscilan entre $21 \%$ y $30 \%$, lo que indica que dentro de las respuestas obtenidas en las encuestas hubo diversos puntos de vista sobre las percepciones que influyen en la productividad laboral. 


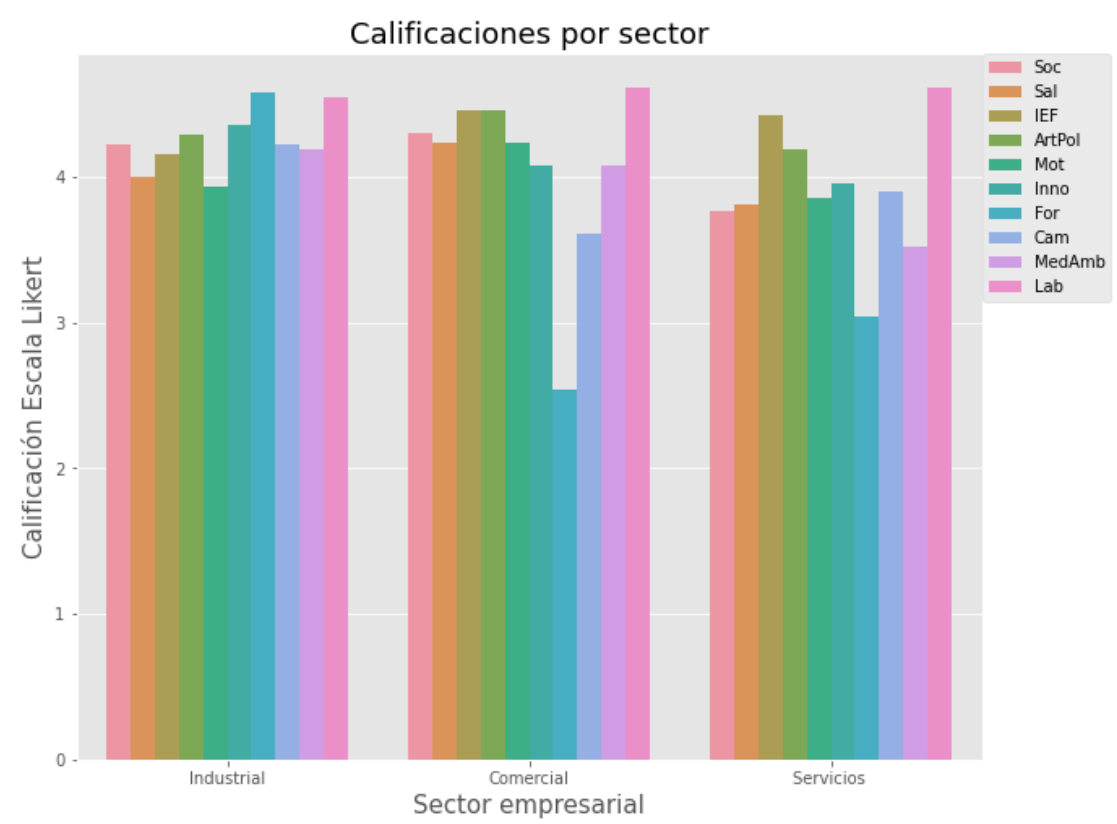

Figura 1. Resultados para cada pregunta de la encuesta agrupadas por variable Figure 3. Results of each item in the survey grouped by variable Fuente: elaboración propia.

Tabla 4. Resumen de estadísticas de las variables o categorías encuestadas Table 4. Summary of statistics of the variables or categories in the survey

\begin{tabular}{|c|c|c|c|c|c|c|c|c|c|c|}
\hline \multicolumn{11}{|c|}{ Resumen estadísticas } \\
\hline & Soc & Sal & IEF & ArtPol & Mot & Inno & For & Cam & MedAmb & Lab \\
\hline Cuenta & 65 & 65 & 65 & 65 & 65 & 65 & 65 & 65 & 65 & 65 \\
\hline Media & 4.09 & 3.98 & 4.31 & 4.29 & 3.97 & 4.17 & 3.68 & 4 & 3.95 & 4.58 \\
\hline Moda & 4 & 4 & 5 & 4 & 5 & 5 & 5 & 5 & 4 & 5 \\
\hline Desviación estándar & 0.88 & 0.78 & 0.86 & 0.7 & 1 & 1.08 & 1.39 & 1.13 & 0.94 & 0.66 \\
\hline Min & 2 & 2 & 2 & 3 & 1 & 1 & 1 & 1 & 2 & 2 \\
\hline Cuartil $25 \%$ & 4 & 4 & 4 & 4 & 3 & 4 & 3 & 3 & 3 & 4 \\
\hline Cuartil $50 \%$ & 4 & 4 & 5 & 4 & 4 & 4 & 4 & 4 & 4 & 5 \\
\hline Cuartil 75\% & 5 & 5 & 5 & 5 & 5 & 5 & 5 & 5 & 5 & 5 \\
\hline Máx & 5 & 5 & 5 & 5 & 5 & 5 & 5 & 5 & 5 & 5 \\
\hline $\begin{array}{l}\text { Coeficiente de } \\
\text { variación }\end{array}$ & 0.22 & 0.2 & 0.2 & 0.16 & 0.25 & 0.26 & 0.38 & 0.28 & 0.24 & 0.14 \\
\hline
\end{tabular}

Fuente: elaboración propia.

En la Figura 4, los gráficos de apilamiento muestran un comportamiento generalizado de las variables, donde su mediana se ubica en 4; 5 variables presentan valores considerados atípicos o fuera de la distribución. Estos valores se ubican en calificaciones de 2 y 1, respectivamente. En general, el promedio de los datos es superior al cuartil $50 \%$. 


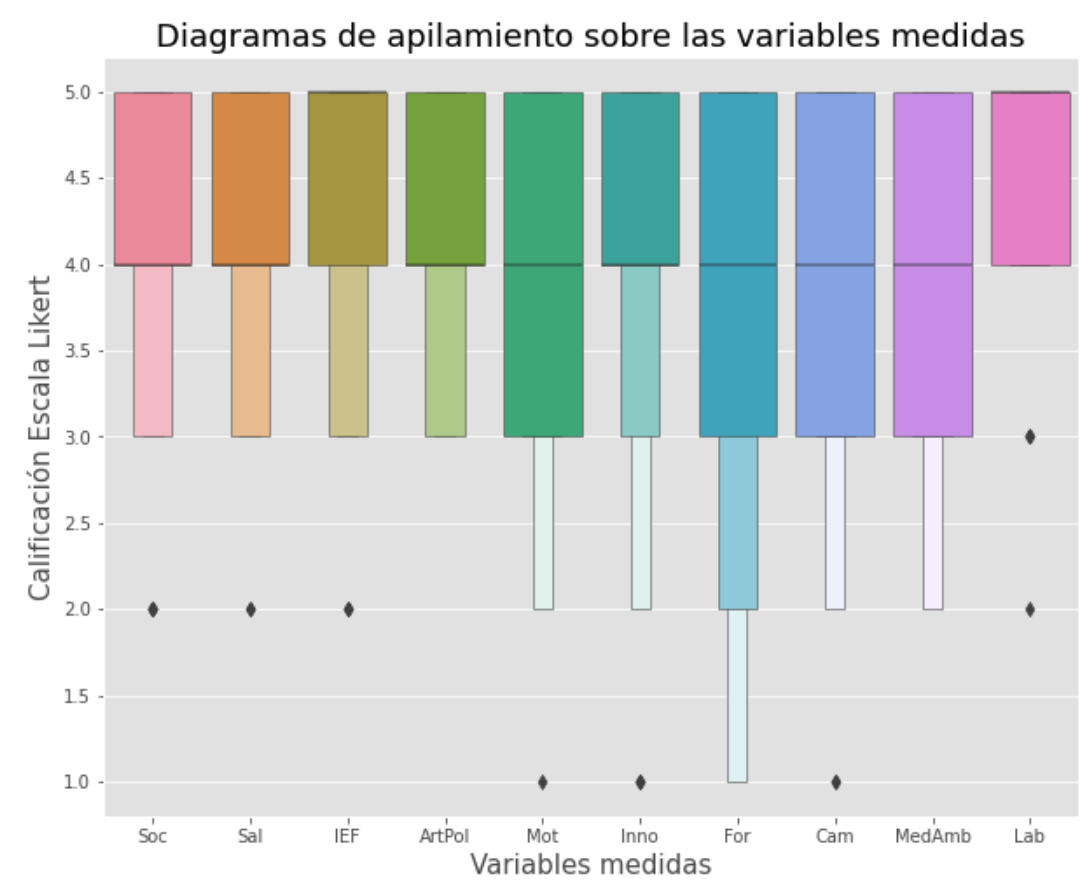

Figura 4. Gráfico de apilamiento por variable o categorías

Figure 4. Boxen plot of variables or categories Fuente: elaboración propia.

Para identificar la existencia de relaciones lineales entre pares de variables en la medición de la productividad, se utilizó un mapa de calor para indicar la presencia de correlaciones. La Figura 5 presenta la matriz de Spearman entre cada par de variables de la encuesta para la medición de la productividad. Estas variables muestran la fuerza de las relaciones lineales medidas a través del coeficiente de correlación, donde los cuadros con mayor tonalidad presentan valores cercanos a 1 , que identifica una correlación lineal positiva entre factores (Uribe Gómez, 2021). La matriz de correlación muestra que la motivación y la adaptabilidad al cambio tienen un coeficiente de correlación positivo y alto, al igual que la innovación y la adaptabilidad al cambio. Por otro lado, la formación de personal cuenta con menos cantidad de relaciones lineales con las otras variables del estudio, en particular con aspectos sociales, remuneración salarial, articulación de políticas y apoyo gubernamental y laboral, esto debido a su resultado de correlación cercano a cero; adicional, el signo negativo en presente en el coeficiente entre la variable de formación y los aspectos sociales indica una relación inversa. 


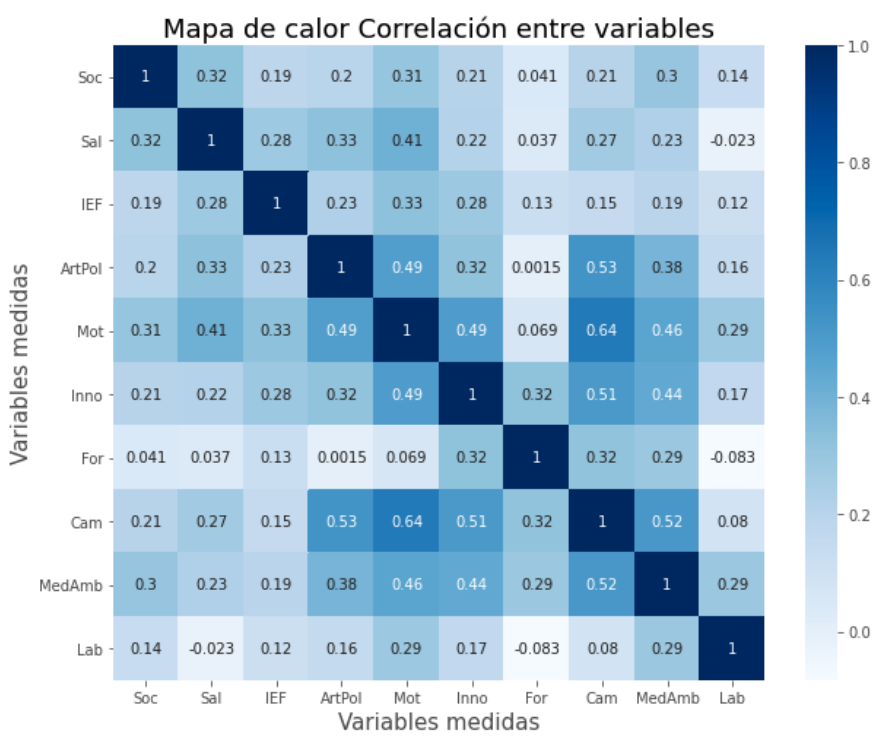

Figura 5. Matriz de correlaciones entre variables o categorías

Figure 5. Matrix of correlations between variables or categories Fuente: elaboración propia.

La Figura 6 muestra los gráficos de barras para cada variable del estudio, donde se puede observar, en general, que las distribuciones tienen una simetría hacia la derecha, es decir hacia valores con calificaciones de 4 y 5 . Con excepción de los aspectos sociales, la remuneración salarial y la articulación de políticas y apoyo presentan un comportamiento menos sesgado y más centrado. La Figura 7, por su parte, presenta la distribución total de resultados de encuestas Likert donde se presenta el panorama general para todas las calificaciones, en esta se puede visualizar en la gran mayoría de los datos que se centran entre 5 y 4; por otro lado, se encuentra que los resultados con calificaciones de 3, 2 y 1 obtienen el menor porcentaje del total.

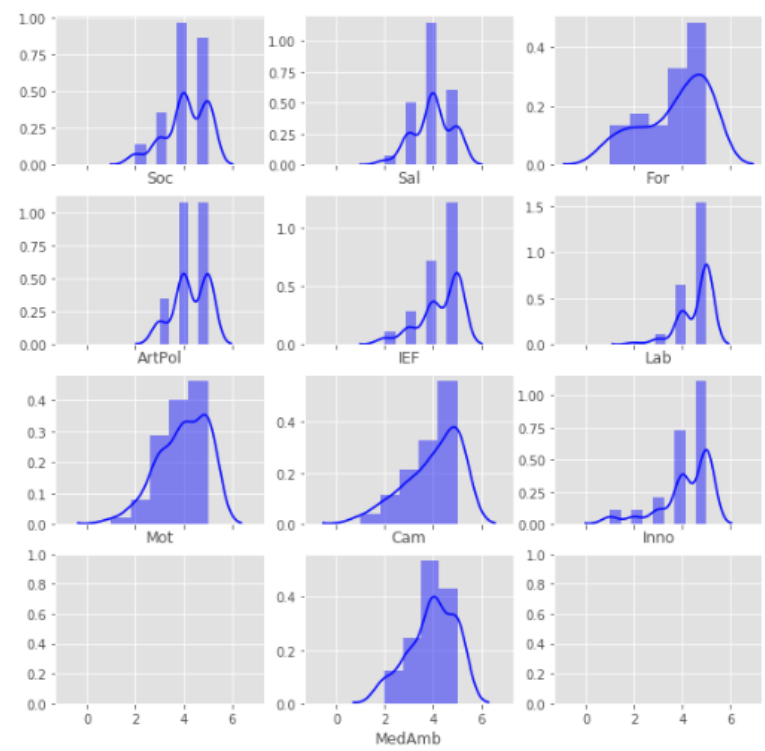

Figura 6. Gráficos de barra de la distribución de los datos totales de la encuesta Figure 6. Bar charts of the distribution of all the data collected in the survey Fuente: elaboración propia. 


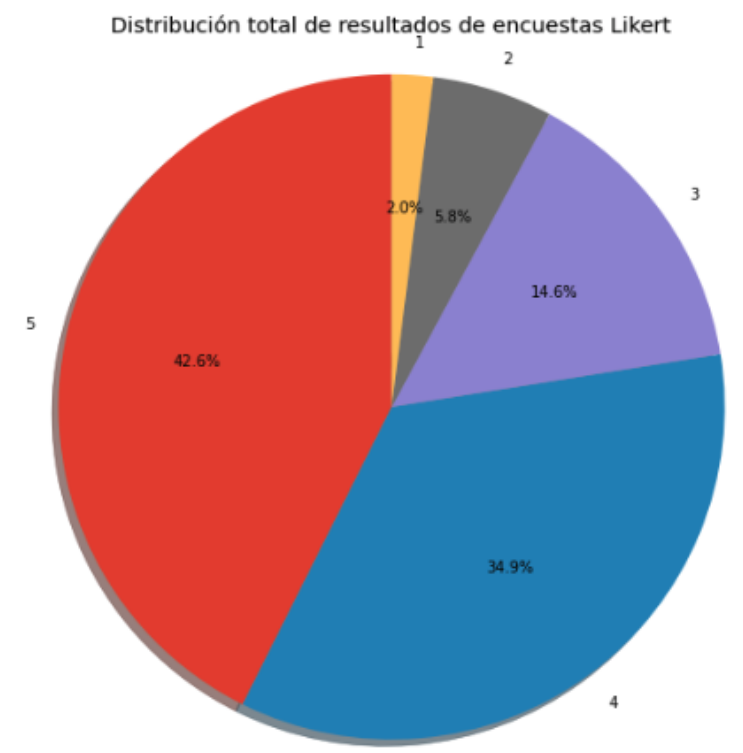

Figura 7. Distribución de los datos totales de la encuesta

Figure 7. Distribution of all the data collected in the survey Fuente: elaboración propia.

La Tabla 5 muestra las estadísticas básicas para el total de resultados de la encuesta, lo que complementa los análisis previos para analizar el comportamiento de los datos, en este caso, el coeficiente de variación es de $24 \%$, esto implica que los datos encontrados no son homogéneos y presentan una consistencia débil, ya que como lo indican Bindu et al. (2019), este tipo de conclusiones sobre los datos se encuentra cuando el coeficiente de variación está entre un $15 \%$ y $33 \%$, asimismo, Paixão et al. (2020) consideran que valores de coeficientes de variación entre $21 \%$ y $30 \%$ son altos, significando así una alta dispersión; también se observa una desviación estándar de 0.99, lo que indica que alrededor de la media hay una dispersión de casi 1 unidad de calificación. El rango de los datos y sus valores máximos y mínimos muestran, de igual forma, una amplia dispersión. El valor positivo de la curtosis muestra que hay un mayor valor de los datos en torno a la media de 4.10 .

Tabla 5. Estadísticas básicas para el total de los datos Table 5. Basic statistics of all the data

\begin{tabular}{lc}
\hline Media & 4.101 \\
\hline Error típico & 0.038 \\
\hline Mediana & 4 \\
\hline Moda & 5 \\
\hline Desviación estándar & 0.994 \\
\hline Varianza de la muestra & 0.988 \\
\hline Curtosis & 0.896 \\
\hline Coeficiente de asimetría & -1.110 \\
\hline Rango & 5 \\
\hline Mínimo & 0 \\
\hline Máximo & 5 \\
\hline Suma & 2666 \\
\hline Cuenta & 650
\end{tabular}


La Tabla 6 presenta los resultados porcentuales de las calificaciones positivas que recibió cada ítem; en general, la estabilidad laboral, la comunicación, la innovación y los salarios son variables con mayor calificación positiva relacionada con la productividad; en cambio, la capacitación y la infraestructura no son aspectos significativos para la medición.

Tabla 6. Resumen hallazgos encuestas a los empleados

Table 6. Summary of findings from employee responses

\begin{tabular}{lc}
\hline Respuestas & Respondió (\%) \\
\hline Hay estabilidad laboral & $94.66 \%$ \\
\hline Hay buena comunicación con los jefes & $88 \%$ \\
\hline La organización es innovadora & $84 \%$ \\
\hline Los salarios son benéficos & $81.33 \%$ \\
\hline El entorno social es seguro & $78.66 \%$ \\
\hline Están motivados con las actividades en la & $73.33 \%$ \\
\hline organización & $73.33 \%$ \\
\hline trabajadores & $72 \%$ \\
\hline Existe la cultura ambiental en la organización & $66.66 \%$ \\
\hline Tuvieron procesos de capacitación & $60 \%$ \\
\hline Tiene una infraestructura adecuada & Fuente: elaboración propia.
\end{tabular}

En términos generales, la medida de los 10 factores que representan la productividad tiene altas dispersiones, es decir, existen simultáneamente puntos de vista con calificaciones altas y también bajas, esto se observa en las estadísticas para cada variable y por el conjunto total de datos. Sin embargo, existen variables que, de acuerdo con la literatura y debido al comportamiento obtenido a partir de las encuestas, representan bien a la variable productividad.

\section{DISCUSIÓN}

De acuerdo con los resultados encontrados en el análisis propio de la información suministrada, a través de la encuesta realizada se pudo percibir que son varios los aspectos asociados con la productividad que se han evaluado positivamente como generadores de oportunidades y mejoras en la productividad; en promedio, las variables infraestructura física, articulación de políticas y apoyo gubernamental, innovación, investigación y avance tecnológico y laboral contienen las calificaciones más alta en los sectores productivos. Considerando los aspectos teóricos evaluados se percibe que, efectivamente, la innovación se relaciona dentro del concepto de productividad a través de sus procesos. Por otro lado, desde la economía, los referentes teóricos destacan que la estabilidad laboral brinda al interior de la organización un aspecto importante en el logro de mejoras en la productividad (Bril et al., 2021). Ahora, ¿qué permite al trabajador obtener estabilidad laboral? Básicamente el tipo de contratación que pueda tener frente a la organización, pues existen contratos variables sujetos a los niveles de producción denominados por servicios o labor, donde este documento le exige al trabajador alta producción porque su salario depende de los niveles de eficiencia y eficacia desarrollados, situación que le permite a la organización disminución de sus costos fijos al 
convertirlos en variables; por otra parte, hay contratos fijos, y como lo indican Trajtembertg y Varela (2015), que son de tiempo indeterminado o sin plazo de finalización, los cuales brindan una mejor estabilidad al asalariado. Con lo anterior hay una dicotomía para la organización y a la vez para los trabajadores, y es obtener productividad con contratos que brinden estabilidad laboral.

De igual forma, se encuentra desde teóricos como Mary Parker y Elton Mayo, donde la comunicación y la articulación de políticas al interior de la organización son factores importantes a la hora de considerar la productividad, y en la que la comunicación en la organización es «...un tema transversal que atraviesa otros fenómenos organizacionales como el liderazgo, el trabajo en equipo, la generación y transferencia de conocimiento o el cambio» (Pepe, 2012, p. 29). Desde esa perspectiva, es la comunicación en cualquier organización el vínculo y el principal medio de lograr niveles de socialización, y es la premisa principal para obtener los objetivos organizacional incluyendo la productividad; sin embargo, se podría considerar grosso modo que los espacios con buena infraestructura física armonizan el ambiente, permiten accesibilidad, movilidad, amplitud, "son numerosos los elementos y condicionantes del espacio de práctica: el tamaño, la acústica, la temperatura, la ventilación, la iluminación, el drenaje y limpieza de las pistas, el mobiliario, la accesibilidad al material, y la contaminación ambiental» (Hill y Hulbert, 2007; Gil et al., 2010; González-Calvo et al., 2018, citados por Bores-García et al., 2020, p. 44).

Sorprendentemente, el salario y la formación cuentan con bajas calificaciones entre los encuestados, a pesar de la importancia que, desde la concepción teórica, destacan Taylor y Gantt. Ahora, con respecto al salario, Jiménez Montero (2010) describe en ser acorde para la organización y el trabajador, el cual debe contener un componente fijo que le permita mantener unas necesidades básicas satisfechas al trabajador, y una parte variable que a su vez le haga participe de los resultados financieros de la organización, esta última apunta, básicamente, al concepto de productividad en su gran mayoría. El salario es un tema de amplia discusión para la organización y los trabajadores, el cual, desde lo propuesto en este estudio, debe fundamentarse bajo condiciones de productividad de tal manera que genere bienestar y no simplemente un instrumento generador de plusvalía. Del tema de la formación, pese a no tener un reconocimiento por parte del trabajo de campo desarrollado, Sánchez (1997) lo indica como el vehículo idóneo para transmitir y llevar a la práctica cualquier saber. En ese sentido, el tema de la formación en cualquier organización es de suma importancia, pues es pasar de lo empírico y tradicional a formas elaboradas y documentadas.

De igual manera, la motivación está documentada, a través de Maslow, como la posibilidad de un colaborador de ser productivo; no obstante, en el promedio de calificaciones, se presenta y afecta, en poca forma, la productividad. Pese a lo anterior, Franco-López et al. (2017), citado por FrancoLópez et al. (2020), indicó la investigación y probó que las personas que experimentan mayor bienestar psicológico son aquellos que orientan sus motivaciones (extrínseca e intrínseca) a mayores logros; en ese sentido, es menester que las organizaciones que busquen mejores resultados, entre ellas tener productividad alta, deben tener en sus colaboradores estrategias que faculten a mantener motivación de las actividades laborales.

Asimismo, no se destaca la participación medio ambiental y la conciencia ambiental en las percepciones de los empleados frente a la productividad; en igual proporción, no existe una teoría concluyente que indique lo mencionado anteriormente y, desde los resultados prácticos, este factor, en promedio, ha sido considerado como el de menor calificación. No obstante, la variable medio 
ambiente se destaca por ser una categoría con una cantidad de vínculos lineales con otras variables, lo que indicaría que aumentar o mejorar este aspecto impactaría positivamente en otras variables.

\section{CONCLUSIONES}

La productividad es un aspecto fundamental para todo el tejido empresarial y el mercado en general, es el soporte del crecimiento de cualquier organización y toda aspiración inicial que debe tener una unidad productiva. Pero ¿cómo lograrlo? Es una pregunta que tiene tantas aristas como organizaciones haya, porque no existen fórmulas únicas que permitan su aplicabilidad con iguales resultados; ahora bien, tratando de cotejar los aspectos teóricos, la problemática encontrada en el área de análisis y los hallazgos, se pueden argumentar varios elementos importantes:

El trabajo de investigación conduce a varias reflexiones, entre ellas que mejorar las percepciones y las valoraciones dadas por los empleados permitirá lograr una mayor oferta agregada al mercado, siempre y cuando se ligue al concepto de sostenibilidad; por esto, en el momento histórico actual, la pertinencia del factor ambiental en los procesos productivos debe ser prioritario; lograr productividad con sostenibilidad debe ser el paradigma de todos los estamentos de la sociedad, desde la política económica del Estado, las sanas costumbres en el consumo y el aporte que deben hacer las organizaciones productivas. Es reflexionar y actuar para las generaciones futuras. Es de destacarlo porque esto denota niveles de conciencia ambiental, pensado desde la difícil situación que afronta el mundo por el agudo deterioro del medio ambiente. Sumamos, además, la prioridad de involucrar a todas las fuerzas sociales, y los planteamientos de Petty, Malthus, McGregor en la teoría Y, cuando destacan que son la formación y la educación las que deben preparar a toda persona para ser productiva, lográndose solo a través de una formación holística, integral, donde prime el factor ambiental. Lo anterior, dentro del trabajo de investigación, presentó solo una relación lineal con otras variables incluyendo la productividad.

Un factor decisivo obtenido en los resultados, con respecto de la productividad, es la investigación, porque solo así se genera innovación, nuevas ideas que desarrollan productos para incrementar la productividad. Existen cuatro tipos de procesos innovadores: productos, proceso, comercialización y organizativa. Para tener alta productividad, las organizaciones deben ser innovadoras. En la matriz de correlación, los factores innovación, investigación y avance tecnológico fueron importantes en los cuatro procesos, aunque el coeficiente de variación para cada uno tuvo gran dispersión. Con base en esto, cabe destacar que en organizaciones que buscan productividad vía innovación, el elemento central es la gestión del conocimiento, donde los procesos de aprendizaje que se construyan logren socializarse y formen parte de la cultura organizacional.

Otra situación, no tan relevante en los hallazgos, pero que amerita un reconocimiento por ser un aspecto que va insertado en la parte psicológica de los seres humanos, es la motivación, definida esta como impulsos, deseos, necesidades, anhelos y fuerzas similares que tienen su origen en el entorno cultural. Estos impulsos median en la forma como una persona interpreta su realidad frente a su trabajo, y en la forma en cómo orienta su vida personal. La motivación, como factor de la productividad, arrojó en la matriz de correlación un vínculo con seis factores, lo cual lo hace destacable. Según la literatura, se destacan dos tipos de motivación en el ser humano: factores intrínsecos, los cuales nacen al interior de la persona; y factores extrínsecos, que son aquellos que se 
encuentran en el entorno. Los primeros prevalecen y son estados emotivos que cada persona tiene; en ellos se destaca la vocación, el reconocimiento y la aceptación, son de larga duración y se convierten en la «chispa» que mantiene viva la «llama». Ahora bien, los factores extrínsecos generan efectos de menor duración en la motivación, tal es el caso de los salarios y la infraestructura, entre otros. Toda organización debe velar por un talento humano motivado, dispuesto al cumplimiento de las tareas, pues esto lo hace altamente productivo (visión humanística de Mary Parker). Es recomendable, desde la perspectiva humanística de Elton Mayo, desarrollar en la organización relaciones que posibiliten espacios a un clima laboral ajustado a las condiciones del talento humano.

Por su parte, el salario representa para el trabajador un elemento extrínseco de la motivación y una de las variables o categorías importante. Para el empresario es un costo o gasto ineludible, pero siempre ligado al desarrollo de la productividad. Aun así, entre la lógica frente al salario (nominal y real) del empresario y del trabajador existe, obviamente, una puja entre empresario y trabajadores, entre ser alto o bajo. Como efecto en la productividad, el salario solo es de corto plazo, pero es un motivador que atrae a la fuerza trabajadora para migrar o mantenerse en un contexto social, así lo confirma la matriz de correlación y la teoría de Taylor. El salario, como factor de productividad, se debe relacionar a un elemento de política económica, como es la estabilidad o nivel general de los precios. En vano sería un salario nominal alto con alta inflación. Cuando se presenta este fenómeno ocurre una disminución en la productividad, pero viene un cuestionamiento, sabiendo que el salario representa un egreso para el empresario. ¿Cuál es la productividad óptima que debe tener la organización para compensar los costos y obtener beneficios? Son posiciones que amparan cualquier investigación que quiera darle salidas al tema de los salarios.

Por último, como aspecto fundamental para las organizaciones, es la noción de cambio como constante en la historia. "Si la organización no cambia, el cambio, la cambia». Es capacidad de adaptación, de leer un entorno exigente sumido en unas dinámicas de alta movilidad, inestabilidad e incertidumbre. El cambio exige capacidad de respuesta inmediata. Los productos, procesos, entendimiento, tecnología y muchos otros aspectos se mantendrán vigentes cuando se adapten permanentemente. En Deming, la visión para una mejora constante es la redefinición constante, mientras que en Juran la calidad se asegura a gran velocidad y los procesos productivos lograrán mantenerse vigentes cuando el empresario logre eficiencia y eficacia en un sentido dinámico.

Es importante resaltar que una de las limitaciones encontradas en la construcción de esta investigación se debe al hecho de utilizar el muestreo no probabilístico, esto impacta en la generalización de los resultados, ya que la muestra en las organizaciones y empleados tiene características propias que deben ser analizadas con mayor detalle; de todos modos se espera en posteriores investigaciones utilizar soportes muestrales más robustos que respondan a la generalización de los resultados y a efectos más concluyentes en los resultados de la investigación.

\section{CONFLICTOS DE INTERÉS}

Los autores declaran que no presentan conflictos de interés financiero, profesional o personal que pueda influir de forma inapropiada en los resultados obtenidos o las interpretaciones propuestas. 


\section{CONTRIBUCIÓN DE AUTORES}

Para el desarrollo de este proyecto todos los autores han realizado una contribución significativa especificada a continuación:

Jorge Ariel Franco-López: revisión de la literatura científica, marco teórico, redacción y ajustes a las distintas revisiones.

Julián Alberto Uribe-Gómez: metodología, análisis de la información y ajustes a las distintas revisiones.

Sebastián Agudelo-Vallejo: trabajo de campo, revisión de la literatura.

\section{REFERENCIAS}

Alonso Villa, P., Juste Carrión, J. (2018). El sector de la automoción en Castilla y León. 50 años de crecimiento económico y productividad: 1961-2011. Revista de Estudios Regionales, n. $113,101-136 . \underline{\text { URL }}$

Álvarez Pinilla, A. (2001). La medición de la eficiencia y la productividad. Ediciones Pirámide.

Alvarez, R. (2015). Productivity, Trade, and Development in Latin America. Emerging Markets Finance and Trade, v. 51, n. 1, 1-2. https://doi.org/10.1080/1540496X.2015.998058

Área Metropolitana del Valle de Aburrá. (2010). Centralidad Sur. Antioquia. Medellín: Litografía Dinámica.

Arias, F. (2011, 22 de noviembre). Centralidad Sur, futuro polo de emprendedores. El Colombiano. $\underline{\mathrm{URL}}$

Ayoví Ramírez, M. W., La Serna Palomino, N. B. (2021). Impacto de gestión de la innovación y la gestión estratégica en la productividad de las pymes de TIC: estudio empírico. Revista Ibérica de Sistemas e Tecnologias de Informação, n. E39, 569-585. URL

Baptista, A. (2005). El capitalismo rentístico: elementos cuantitativos de la economía venezolana. Cuadernos de Cendes, v. 22, n. 60, 97-115. URL

Bergston, E. L., Haapakangas, A., Larsson, J., Jahncke, H., Hallman, D. M. (2021). Effects of relocation to activity-based workplaces on perceived productivity: Importance of changeoriented leadership. Applied Ergonomics, v. 93, 103348.

https://doi.org/10.1016/i.apergo.2020.103348

Bindu, K. H., Raghava, M., Dey, N., Rao, C. R. (2019). Coefficient of variation and machine learning applications (1a ed.). https://doi.org/10.1201/9780429296185 
Bores-García, D., Marín-Rojas, A. L., Polo-Recuero, B. (2020). La influencia del espacio físico en el proceso de enseñanza-aprendizaje de la educación física. Revista Estilos de Aprendizaje, v. 13, n. $25,42-54$. URL

Bragg, M., Arshonsky, J., Pageot, Y., Eby, M., Tucker, C., Yin, S., Jay, M. (2021). Student-led research team-building program may help junior faculty increase productivity in competitive biomedical research environment. BMC Medical Education, v. 21, n. 3. https://doi.org/10.1186/s12909-020-02396-8

Bril, A., Kalinina, O., Valebnikova, O., Valebnikova, N., Camastral, M., Shustov, D., Ostrovskaya, N. (2021). Improving Personnel Management by Organizational Projects: Implications for Open Innovation. Journal of Open Innovation: Technology, Market, and Complexity, v. 7, n. 2, 105. https://doi.org/10.3390/joitmc7020105

Bruce, P., Bruce, A., Gedeck, P. (2020). Practical statistics for Data scientists. (2nd ed.). O'Reilly Media.

Bušelić, M., Pavlišić, P. (2016). Innovations As an Important Factor Influencing Labour Productivity in the Manufacturing Industry. Ekonomski vjesnik: Review of Contemporary Entrepreneurship, Business, and Economic Issues, v. 29, n. 2, 405-420. URL

Centro de Ciencia y Tecnología de Antioquia. (s.f.). Enplanta 2020. Fortalecimiento de la productividad para la recuperación del tejido empresarial.

Cimoli, M., Porcile, G., Primi, A., Vergara, S. (2005). Cambio estructural, heterogeneidad productiva y tecnología en América Latina. En Cimoli, M. (ed.) Heterogeneidad estructural, asimetrías tecnológicas y crecimiento en América Latina. Nacionaes Unidas. URL

Clavijo, S. (2003). Crecimiento, productividad y la nueva economía: implicaciones para Colombia. 1-37. URL

Dangeti, P. (2017). Statistics for Machine Learning. Packt Publishing Ltd.

de la Cuesta González, M., Pardo Herrasti, E., Paredes Gázquez, J. D. (2015). Identificación de indicadores relevantes del desempeño RSE mediante la utilización de técnicas multicriterio. Innovar: Revista de Ciencias Administrativas y Sociales, v. 25, n. 55, 75-88. https://doi.org/10.15446/innovar.v25n55.47197

de Vries, G., Jiang, A., Lemmers, O., Wei, S. (2021). Firm productivity and functional specialisation. The World Economy, v. 44, n. 5, 1232-1260. https://doi.org/10.1111/twec.13055

Delfín Ortega, O. V., Navarro Chávez, J. C. L. (2020). La productividad de los puertos en la región del APEC: un estudio a través del análisis de la frontera estocástica. Contaduría y Administración, v. 66, n. 1, 1-34. https://doi.org/10.22201/fca.24488410e.2021.1998

Fau, M. E. (2000). Adam Smith. Clásicos resumidos (1ra ed.). La Bisagra Editorial. 
Franco-López, J. A., López-Arellano, H., Arango-Botero, D. (2020). La satisfacción de ser docente: un estudio de tipo correlacional. Revista Complutense de Educación, v. 31, n. 1, 55-67. https://doi.org/10.5209/rced.61775

Galeano, M. E. (2004). Diseño de proyectos en la investigación cualitativa. Fondo Editorial Universidad Eafit.

Gómez Muñoz, W. A., Posada Posada, C. E., Rhenals Monterrosa, R. (2019). Determinantes de la productividad multifactorial: los casos de las principales economías latinoamericanas y emergentes de Asia (1960-2015). Centro de Investigaciones en Economía y Finanzas. http://hdl.handle.net/10495/10399

Gori Maia, A., Sakamoto, A. (2018). Does wage reflect labor productivity? A comparison between Brazil and the United States. Brazilian Journal of Political Economy, v. 38, n. 4, 629-649. $\underline{U R L}$

Guzmán Soria, E., de la Garza-Carranza, M. T., García Salazar, J. A., Hernández Martínez, J., Rebollar Rebollar, S. (2020). Análisis de productividad y eficiencia de las MIPYMES en México. Revista Globalización, Competitividad y Gobernabilidad, v. 14, n. 2, 69-83. URL

Hinojo Lucena, F. J., Aznar Díaz, I., Romero, J. M. (2020). Factor humano y productividad empresarial: un enfoque desde el análisis de competencias transversales. Innovar, v. 30, n. 76, 51-62. https://doi.org/10.15446/innovar.v30n76.85194

Hofman, A., Mas, M., Aravena, C., Fernández de Guevara, J. (2017). Economic Growth and Productivity in Latin America: LA-KLEMS. El Trimestre Económico, v. 84, n. 334, 259-306. https://doi.org/10.20430/ete.v84i334.302

Jensen, P. A., van der Voordt, T. (2020). Productivity as a value parameter for FM and CREM. Facilities, v. 39, n. 5-6, 305-320. https://doi.org/10.1108/F-04-2020-0038

Jiménez Montero, D. (2010). Pautas para la definición de salarios. Revista de Ciencias Económicas, v. 28, n. 2, 393-404. URL

Jurado Jurado, J. C. (2015). Hacia una arqueología de la administración. Cuadernos de Administración, v. 28, n. 50, 113-136. https://doi.org/10.11144/Javeriana.cao28-50.haam

Kamble, R., Wankhade, L. (2017). Perspectives on productivity: identifying attributes influencing productivity in various industrial sectors. International Journal of Productivity and Quality Management, v. 22, n. 4, 536-566. https://doi.org/10.1504/IJPQM.2017.087868

Khan, T., Nouri, H., Booij, M. J., Hoekstra, A. Y., Khan, H., Ullah, I. (2021). Water Footprint, Blue Water Scarcity, and Economic Water Productivity of Irrigated Crops in Peshawar Basin, Pakistan. Water, v. 13, n. 9, 1249. https://doi.org/10.3390/w13091249 
Kim, J., Park, J. (2018). The role of total factor productivity growth in middle-income countries. Emerging Markets Finance and Trade, v. 54, n. 6, 1264-1284. https://doi.org/10.1080/1540496X.2017.1422244

Koontz, H., Weihrich, H., Cannice, M. (2012). Administración: una perspectiva global (14a ed.). Mc Graw Hill.

Krizanic, F., Hodzic, S., Vojinovic, B. (2021). Fiscal Policy, Factors of Endogenous Growth and Dynamics of Slovenian Exports. Engineering Economics, v. 32, n. 1, 27-34. https://doi.org/10.5755/j01.ee.32.1.26311

Laborde, M. N., Veiga, L. (2011). La productividad. IEEM Revista de negocios, n. 6, 68-69. URL

Maldonado, N., Sánchez, E. (2012). Rutas de transformación productiva. Cuadernos de economía, v. 31, n. $57,113-145 . \underline{U R L}$

Marquetti, A. (2004). Do rising real wages increase the rate of labor-saving technical change? Some econometric evidence. Metroeconomica, v. 55, n. 4, 432-441. https://doi.org/10.1111/j.1467-999X.2004.00201.x

Mayol Marcó, D. D. (2013). Taylor, cien años después. Revista Venezolana de Análisis de Coyuntura, v. 19, n. 2, 195-209. URL

McNamara, T., Meloso, D., Michelotti, M., Puncheva-Michelotti, P. (2021). 'You are free to choose ... are you?' Organisational punishment as a productivity incentive in the social science literature. Human Relations. https://doi.org/10.1177/00187267211007891

Medina Fernández de Soto, J. E. (2009). Modelo integral de productividad: una visión. Universidad Sergio Arboleda. URL

Mera Ruiz, C. (2017). El concepto de tiempo en el Taylorismo. Editorial Politécnico Grancolombiano.

Observatorio Aburrá Sur. (2016). Informe Calidad de Vida 2012-2015. URL

Ortiz, C. H., Jiménez, D. M., Cruz, G. N. (2019). El impacto de la infraestructura en el crecimiento económico colombiano: un enfoque smithiano. Lecturas de Economía, n. 90, 97-126. https://doi.org/10.17533/udea.le.n90a04

Paixão, C. S. S., Voltarelli, M. A., da Silva, R. P., Borba, M. A. D. P., Torres, L. S. (2020). Statistical process control applied to monitor losses in the mechanized sugarcane harvesting. Engenharia Agricola, v. 40, n. 4, 473-480. https://doi.org/10.1590/1809-4430-Eng.Agric.v40n4p473-480/2020 
Partridge, M., Tsvetkova, A., Betz, M. (2021). Are the most productive regions necessarily the most successful? Local effects of productivity growth on employment and earnings. Journal of Regional Science, v. 61, n. 1, 30-61. https://doi.org/10.1111/jors.12499

Pepe, A. I. (2012). Comunicación intercultural en la organización. Dixit, n. 17, 28-39. URL

Peralta, M. L., Candanedo, M., Icaza, A., Pinto, B., Lima, M. (2019). Evaluación de los impactos del crecimiento urbano disperso sobre los sistemas de transporte en el área Este de la ciudad de Panamá. En Industry, Innovation, and Infrastructure for Sustainable Cities and Communities: Proceedings of the 17th LACCEI International Multi-Conference for Engineering, Education and Technology. https://doi.org/10.18687/LACCEI2019.1.1.406

Pereira Pérez, Z. (2011). Mixed Method Designs in Education Research: a Particular Experience. Revista Electrónica Educare, v. 15, n. 1, 15-29. https://doi.org/10.15359/ree.15-1.2

Quinde Rosales, V., Bucaram Leverone, R., Saldaña Vargas, M., Ordeñana Proaño, A. (2020). Relación entre crecimiento y desarrollo económico: caso Ecuador. Universidad y Sociedad, v. 12 , n. 3, 60-66. URL

Quintero Arango, L. F., Betancur Arias, J. D. (2018). Modelo de salario emocional para la fidelización de los colaboradores en la búsqueda de una organización competitiva. Revisar y corregir. Revista Espacios, v. 39, n. 41. URL

Ros, J. (2014). Productividad y crecimiento en América Latina: ¿por qué la productividad crece más en unas economías que en otras? Naciones Unidas. http://repositorio.cepal.org/handle/11362/36770

Salcines, J. V., Freire, M. J. (2010). El valor económico de la educación a través del pensamiento económico: Desde el Mercantilismo hasta Alfred Marshall (siglo XIX). Revista de la educación superior, v. 39, n. 153, 53-64. URL

Sánchez Murillo, A. J. (2006). Peter Drucker, innovador maestro de la administración de empresas. Cuadernos Latinoamericanos de Administración, v. 2, n. 2. URL

Sánchez, M. A. (1997). Ética, organización y formación. Reis: Revista Española de Investigaciones Sociológicas, n. 77-78, 185-198. https://doi.org/10.2307/40183943

Seclen-Luna, J. P., Moya-Fernández, P., Pereira, Á. (2021). Exploring the Effects of Innovation Strategies and Size on Manufacturing Firms' Productivity and Environmental Impact. Sustainability, v. 13, n. 6, 3289. https://doi.org/10.3390/su13063289

Sedaghat, M. (2013). A productivity improvement evaluation model by integrating AHP, TOPSIS and VIKOR methods under fuzzy environment (case study: State-owned, partially private and private banks in Iran). Economic Computation and Economic Cybernetics Studies and Research, v. 47, n. 1, 235-258. URL 
Smith, A. D. (2019). Customer Relationships Management for Improved Productivity. International Journal of Adult Vocational Education and Technology (IJAVET), v. 10, n. 2, 40-48. https://doi.org/10.4018/IJAVET.2019040104

Tejedor Estupiñán, J. M. (2013). Relaciones entre la productividad y los salarios en Colombia. Revista Finanzas y Política Económica, v. 5, n. 2, 11-22. https://doi.org/10.14718/revfinanzpolitecon.v5.n2.2013.439

Trajtembertg, D., Varela, H. (2015). Movilidad laboral de los trabajadores con contratos eventuales: ¿mecanismo de inserción en empleos estables o regularizacion de la inestabilidad laboral? Trabajo y sociedad, n. 24, 109-130. URL

Uribe Gómez, J. A. (2021). Fundamentos de control estadístico de procesos para gestores y administradores tecnológicos. Fondo Editorial ITM.

https:/doi.org/10.22430/9789585122352

Valli, V., Saccone, D. (2015). Structural change, globalization and economic growth in China and India. The European Journal of Comparative Economics, v. 12, n. 2, 133-163. https://doi.org/10.25428/1824-2979/201502-133-163

Vázquez López, R. (2021). Productividad laboral y competitividad externa en el sector manufacturero mexicano tras la apertura comercial, 1996-2007. Cuadernos de economía, v. 40, n. 82. https://doi.org/10.15446/cuadecon.v40n82.80510

Vera, L. (2009). Cambio estructural, desindustrialización y pérdidas de productividad: Evidencia para Venezuela. Cuadernos del Cendes, v. 26, n. 71, 89-115. URL

Vera, L. V., Vera, J. A. (2021). Productividad laboral y salario real: relaciones de causalidad en Venezuela. Problemas de desarrollo, v. 52, n. 205, 27-53.

https://doi.org/10.22201/iiec.20078951e.2021.205.69620

Vestby, J., Buhaug, H., Von Uexkull, N. (2021). Why do some poor countries see armed conflict while others do not? A dual sector approach. World Development, v. 138, 105273. https://doi.org/10.1016/j.worlddev.2020.105273

Villalobos Valencia, Á. D., Molero Olivo, L. E., Castellano, A. G. (2021). Análisis de la productividad total de los factores en América del Sur en el período 1950-2014. Lecturas de economía, n. 94, 127-163. https://doi.org/10.17533/udea.le.n94a341253

Villegas Pocaterra, E., Alava Martínez, H. E., Ponce Andrade, J. E., Palacios Molina, D. L. (2020). Productividad total factorial y diferencias de ingreso a nivel internacional: 1950-2017. Revista de Ciencias Sociales, v. 26, n. 3, 327-342. URL

Zhang, Y., Wu, Z. (2021). Intelligence and Green Total Factor Productivity Based on China's Province-Level Manufacturing Data. Sustainability, v. 13, n. 9. https://doi.org/10.3390/su13094989 